\title{
The reality of informed consent: empirical studies on patient comprehension-systematic review
}

\author{
Tomasz Pietrzykowski ${ }^{1}$ and Katarzyna Smilowska ${ }^{2^{*}}$ (D)
}

\begin{abstract}
Background: Informed consent is a basic concept of contemporary, autonomy-based medical practice and facilitates a shared decision-making model for relations between physicians and patients. Thus, the extent to which patients can comprehend the consent they grant is essential to the ethical viability of medicine as it is pursued today. However, research on patients' comprehension of an informed consent's basic components shows that their level of understanding is limited.

Methods: Systemic searches of the PubMed and Web of Science databases were performed to identify the literature on informed consent, specifically patients' comprehension of specific informed consent components.

Results: In total, 14 relevant articles were retrieved. In most studies, few clinical trial participants correctly responded to items that examined their awareness of what they consented to. Participants demonstrated the highest level of understanding (over 50\%) regarding voluntary participation, blinding (excluding knowledge about investigators' blinding), and freedom to withdraw at any time. Only a small minority of patients demonstrated comprehension of placebo concepts, randomisation, safety issues, risks, and side effects.

Conclusions: We found that participants' comprehension of fundamental informed consent components was low, which is worrisome because this lack of understanding undermines an ethical pillar of contemporary clinical trial practice and questions the viability of patients' full and genuine involvement in a shared medical decision-making process.
\end{abstract}

Keywords: Informed consent, Clinical trials, Medical practice, Law, Ethics, Comprehension, Health care quality, Autonomy

\section{Introduction}

Written informed consent (IC) is considered a basic principle of medical practice. It provides information and shares knowledge between the physician and patient and creates a shared-decision-based healthcare plan [1]. In this regard, the IC should implement a principle of autonomy, by which a patient's right to deliberately decide for herself whether to accept or refuse the offered treatment must be respected [2, 3]. However, patients'

\footnotetext{
* Correspondence: kasia.smilowska@gmail.com

${ }^{2}$ Department of Neurology; Centre of Expertise for Parkinson \& Movement Disorders, Radboud University Medical Center, Donders Institute for Brain, Cognition and Behaviour, PO Box 9101, 6500 HB Nijmegen, the Netherlands Full list of author information is available at the end of the article
}

adequate understanding of the provided information is a major limitation.

Within its ethical and legal foundations, the informed consent process is pivotal to supporting ethically sound medical intervention. However, obtaining adequately informed consent from patients is complex because it requires human interactions involving discussion of several elements, such as the patient's condition and therapeutic options, including risks and benefits, inconveniences, and uncertainties. In this regard, IC must include both a form that patients are required to read and sign, and oral communication to ensure adequate

(c) The Author(s). 2021 Open Access This article is licensed under a Creative Commons Attribution 4.0 International License, which permits use, sharing, adaptation, distribution and reproduction in any medium or format, as long as you give appropriate credit to the original author(s) and the source, provide a link to the Creative Commons licence, and indicate if changes were made. The images or other third party material in this article are included in the article's Creative Commons licence, unless indicated otherwise in a credit line to the material. If material is not included in the article's Creative Commons licence and your intended use is not permitted by statutory regulation or exceeds the permitted use, you will need to obtain permission directly from the copyright holder. To view a copy of this licence, visit http://creativecommons.org/licenses/by/4.0/ The Creative Commons Public Domain Dedication waiver (http://creativecommons.org/publicdomain/zero/1.0/) applies to the data made available in this article, unless otherwise stated in a credit line to the data. 
understanding to facilitate voluntary willingness to participate in a clinical trial [4].

Major barriers to adequate IC understanding include the patients' subjective impression that they are well informed and physicians' over-confidence in the intelligibility and quality of the information they provide to patients. Nonetheless, the concept of respecting patients' autonomy in medical research is based on the assumption that the informed consent process actually leads to patients' full comprehension of what they are consenting to. Unless this assumption is demonstrably true, the ethical viability of the current medical experimentation practice is seriously flawed.

Given that most available studies focused on informed consent obtained for the purpose of clinical trials, we limited our scope to this kind of research practice. However, there is no reason to assume that the level of understanding of informed consent granted by patients in a routine medical practice is significantly higher than that in clinical trials. On the contrary, we find it plausible that patients recruited to clinical trials are relatively better informed and physicians may explain the nature of a research intervention and participation conditions more thoroughly. Therefore, it is improbable that patients' actual comprehension of consent in standard medical practice is higher than in the relatively better examined conditions of clinical trials, and there are reasons to expect that it is lower. With this reservation, our conclusions may be extended beyond clinical trial conditions to the more general practice of obtaining informed consent in medical practice.

Therefore, we systematically reviewed the available literature on patients' actual (rather than declared) understanding of what they consented to, with particular interest in questionnaires developed to objectify patients' understanding of the consent content, rather than their subjective impression on how well informed they were during the consenting process, and whether they were satisfied with the way in which their consent was obtained.

\section{Methods}

We performed a systematic review using the Preferred Reporting Items for Systematic Review and Meta-Analysis (PRISMA) criteria [5]. The electronic search to identify and capture informed consent literature was conducted between October 2019 and January 2020. We queried PubMed and Web of Science databases using the following search terms: "informed consent [mh] AND (comprehension [mh] OR perception [mh] OR knowledge [mh] OR decision making [mh] OR understanding OR communication [mh]) AND (randomised controlled trials as topic [mh] OR clinical trial as topic [mh])". No year restrictions were applied. To make the search as comprehensive as possible, we used the Boolean operators "AND" and "OR" to link the search terms.

Inclusion criteria were (a) studies assessing comprehension of IC, (b) English-language articles in peer-reviewed academic/scientific journals, (c) full-text articles available electronically, and (d) articles with available questionnaires used to examine the level of patients' understanding.

Exclusion criteria were (a) studies comparing or evaluating methods of informed consent not related to IC comprehension (defined in inclusion criteria), (b) studies that used intervention to improve patients' understanding, (c) studies that included patients with cognitive decline, (d) qualitative research, (e) articles based on patients' impression of understanding, (f) studies based on interviews, (g) studies that did not provide the questionnaire used, (h) conference abstracts, and (i) animal studies.

We included only articles that examined knowledge about the information included in the IC. In this regard, we excluded articles based on interviews and questionnaires that examined only patients' impressions of understanding (e.g. "Did you receive adequate information about the study?").

Article selection was performed independently by the first (TP) and second (KS) authors. Database searches were completed in a blinded manner using identical search terms. After identifying eligible articles, any doubts were resolved during a meeting to review the queried article(s) against the inclusion and exclusion criteria. The final selection of eligible articles included in the critical appraisals was made based on the agreement between TP and KS.

\section{Results}

\section{Selection process}

The study selection process is shown in Fig. 1. In total, 4263 articles were retrieved from the databases, of which 14 were included in the review based on the inclusion/exclusion criteria (Table 1). The number of participants varied across studies, ranging from 29 [16] to 1835 [9]. In most studies $(n=12)$, participants were adults [7-15, 17-19]; three studies examined parents or guardians $[6,10,16]$; and one study included both adult patients and parents or guardians [10]. Medical specialties included infectious disease in $42 \%(n=6)$, including vaccine studies in $21 \%(n$ $=3)$, oncology in $28 \%(n=4)$; rheumatology in $21 \%(n=3)$; neurology in $7 \%(n=2)$, and others in $7 \%$. Two studies included clinical trials in more than one specialty $[8,15]$. Most studies examined IC-related questions that covered compensation, withdrawal criteria and consequences, study versus treatment, study administration, and randomisation.

\section{Understanding of informed consent}

Questionnaires that examined participants' understanding of IC components included true/false items [9, 10, 18], 


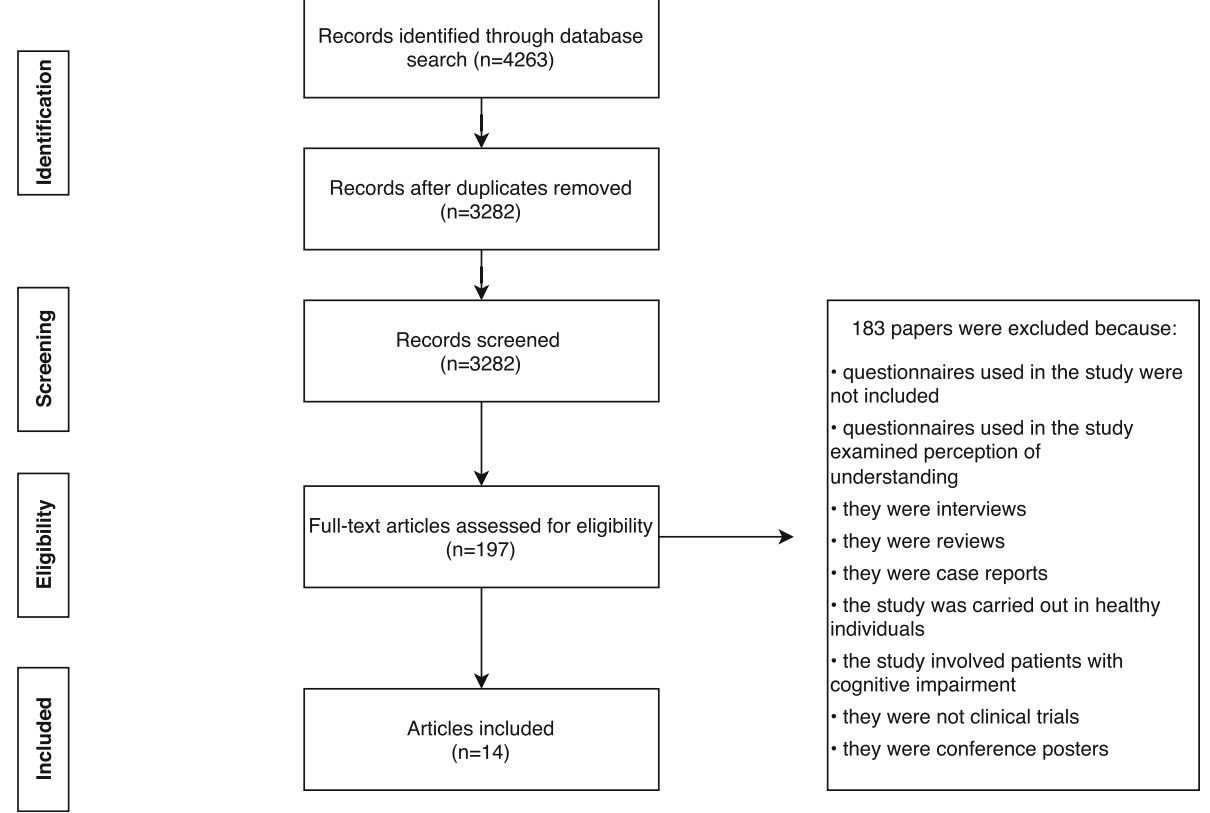

Fig. 1 Selection process for eligible articles

multiple choice items, and the Quality of Informed Consent survey $[7,11]$. Questionnaires differed in the number of items and content. All questionnaires examined participants' recall of IC content, except one, where participants used the IC text to find the answers to the survey questions [19]. Additionally, the elapsed time between participants' experience of the informed consent process and their IC research participation ranged from before the actual IC [18] process to 5 years after the IC [15]; four studies did not report this measure $[8,12,16,19]$.

Three studies examined participants' understanding of the research purpose. Schumacher et al. reported that all participants understood that they were participating in a research study and recognised its purpose [7]. In the remaining two studies, most participants comprehended the study aims $(70-90 \%)[9,19]$.

Voluntary participation was examined in seven studies $[6-9,11,13,14]$. Bergenmar et al. reported the highest level of comprehension, with $96 \%$ of participants comprehending the voluntary nature of their participation [11]. In contrast, Chu et al. reported the lowest level of comprehension, with 53.6\% [8]. Additionally, Krosin et al. noted a significant difference between urban and rural participants, with $85 \%$ and $21 \%$, respectively, showing comprehension of the voluntary nature of participation [13]. Chu et al. reported that $53.6 \%$ patients understood that physicians should not persuade them to participate in a study [8]. Criscione et al. reported that $10 \%$ of participants indicated that their personal doctor would mind if they dropped out of the study [14].
Freedom to withdraw was reported in eight studies $[6-8,10,12,14,17,19]$, which was a relatively wellcomprehended IC component, with the lowest level of $63 \%$ reported by Criscione et al. [14]. Ponzio et al. reported that all participants correctly understood their right to withdraw at any time [19]. Additionally, one study reported on awareness of withdrawal consequences, with $44 \%$ demonstrating comprehension of this point; and withdrawal criteria, with only $10 \%$ showing comprehension [13].

Comprehension of randomisation was investigated in seven studies, with Harrison's study reporting the highest level of understanding (96\%), and Bertoli et al. reporting the lowest $(10 \%)[8,11,12,14-16]$. Similarly, the understating of placebo and active treatment ranged from 13\% [15] to $97 \%$ [9]. Differences by specialty regarding comprehension of the placebo concept were noted by Pope et al., with the lowest comprehension reported in the ophthalmology group (13\%) and the highest in the rheumatology group (49\%) [15].

Risks and benefits were explored in nine [6, 7, 9-11, $13,14,18,19]$ and three studies $[6,7,19]$, respectively. Krosin et al. reported that only $7 \%$ of patients comprehended risks associated with involvement in clinical trials [13]. In contrast, in one group, all patients (who could use the IC text to find questionnaire answers) were aware of potential side effects and risks of the treatment [19]. Ponzio et al. reported the highest between-group differences in the comprehension of study benefits, which ranged from 35.5 to 96.6\%, 


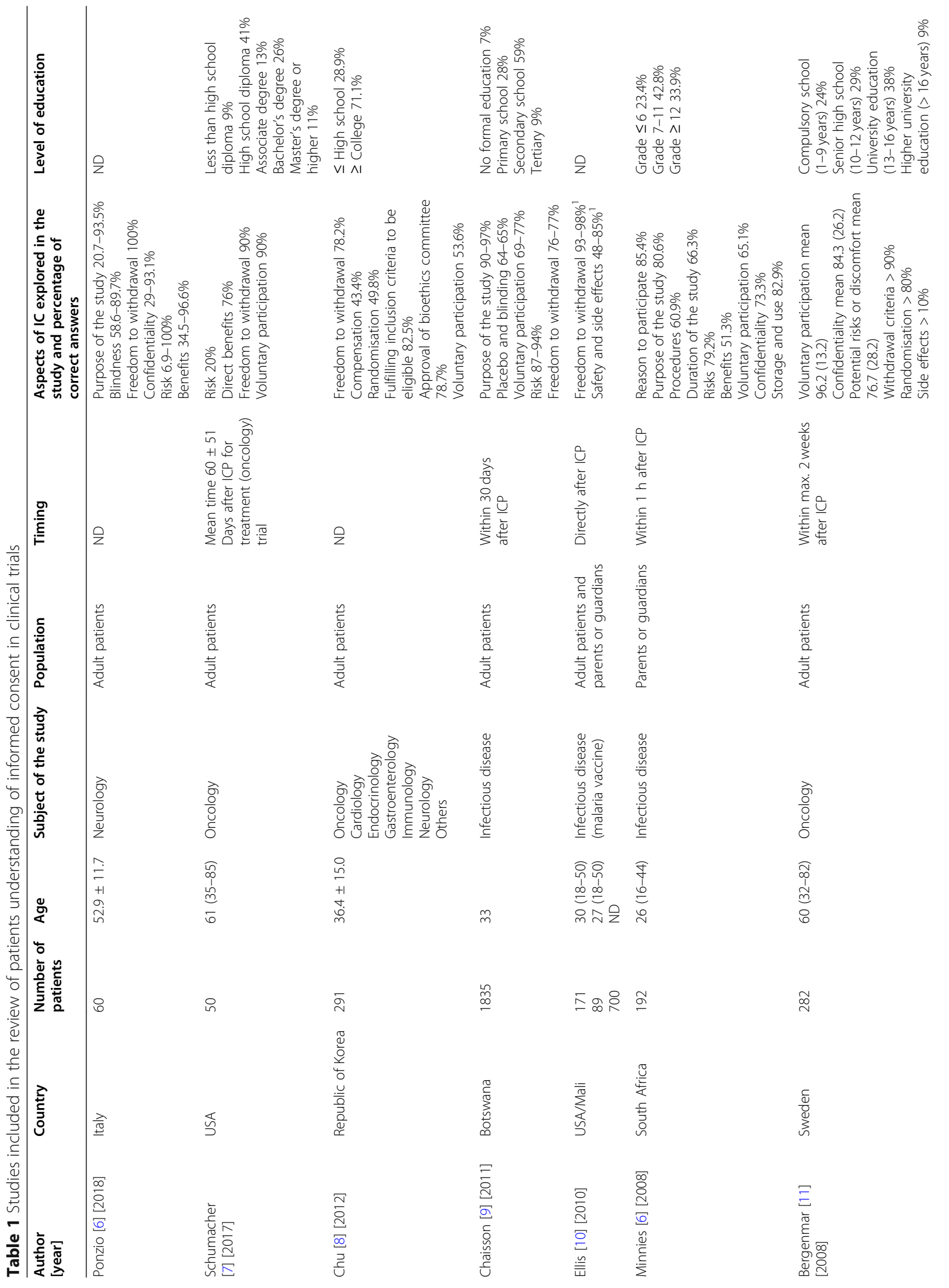




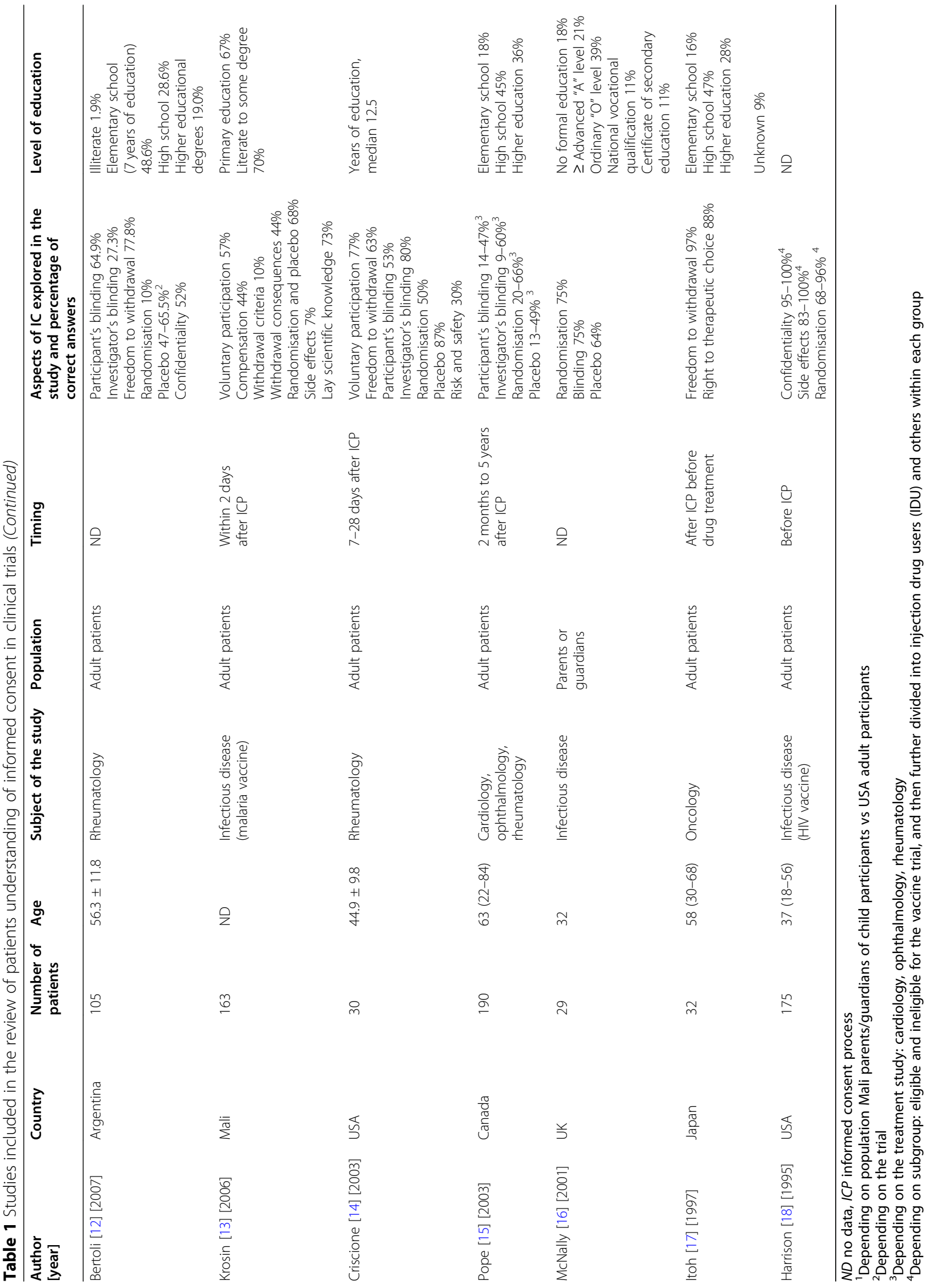


depending on whether participants were successful in finding the answer in the IC text [19].

It is worth noting that Schumacher et al. reported that patients were not aware that the proposed treatment was experimental and not standard therapy [7]. Additionally, only $20 \%$ of participants understood that the benefits of treatment were uncertain and that participation was associated with additional risks. Similarly, over $30 \%$ of patients were not aware that alternative treatments were available.

Furthermore, Chu et al. found that only $43.4 \%$ of patients understood that they would not be reimbursed for all adverse events related to the study. Of note, the authors did not specify the conditions regarding reimbursement. The number of correct responses was higher in the healthy control group than in the patient group (excluding the last question related to reimbursement) [8].

Finally, Bertoli et al. reported that 86 participants $(83.5 \%)$ recalled that they had fully read the informed consent form, while $11.7 \%$ had partially read it and $9 \%$ did not remember to what extent they had read it. Interestingly, most patients (51.4\%) rated their knowledge about the study as high, but objective evaluation of participants' knowledge showed that only $14.3 \%$ demonstrated a high level of knowledge, and $58.1 \%$ and $27.6 \%$ showed intermediate and low knowledge, respectively [12]. Pope et al. reported that $18 \%$ of participants admitted that they had not fully read the study information letter and $10 \%$ admitted that they were afraid to ask questions [15].

\section{Assessment of risk of bias}

Studies included in this review were either randomised nor blinded for the outcomes related to IC. Therefore, the assessment of risk of bias was not possible [20].

\section{Discussion}

We concluded that there are significant discrepancies in research participants' understanding of voluntary participation, blinding, and freedom to withdraw. Only rarely did all participants respond correctly to questionnaire items, indicating that they actually comprehended what they consented to. We found that participants presented the highest level of understanding (over 50\%) about voluntary participation, blinding (excluding knowledge about investigators' blinding), and freedom to withdraw at any time. Further, our results suggest that only a small minority of patients had a clear and accurate understanding of all aspects of their consent. In particular, patients presented significant difficulties in grasping the concept of placebo randomisation, safety, risks, and side effects $[7-16,18,19]$. Additionally, some patients had very limited comprehension of the research benefits $[6,19]$.
Our findings are consistent with the results of previous meta-analyses on the quality of the informed consent process in clinical trials [21]. However, in general, patients included in our review demonstrated lower levels of comprehension. Tam et al. [21] reported that twothirds of participants (the highest reported level) understood the freedom to withdraw from a study at any time, followed by the nature of the study, the voluntary nature of participation, and the potential benefits. In contrast, our results showed that $69.6 \%$ of participants understood the purpose of the study and only $54.9 \%$ could name at least one risk. Finally, approximately half of the participants understood placebo and randomisation concepts. However, in contrast to our review, Tam et al. included data from interviews in their analysis. Surprisingly, in $58.5 \%$ of interviews, participants could not establish whether the interviewers were investigators in the original clinical trial and, as such, could influence the results [21]. In a previous systematic review of clinical trial IC or surgery IC, Falagas et al. concluded that only $50 \%$ of participants properly understood all IC components [22].

These findings demonstrate that crucial information, including risks and benefits, voluntariness, and the relation of trials to standard therapy, are not actually comprehended by a substantial number of participants. This seriously undermines the present practice of providing a sound ethical basis for experimenting with human subjects. Moreover, it seems that patients' understanding of specific IC components has not changed over the past 20 years [21]. The guidelines for good clinical practice in trials, introduced by the World Health Organization 20 years ago, have not affected patients' comprehension [21, 23].

It is natural to expect a correlation between general health literacy and comprehension of information relevant to informed consent. The extent to which deficits in understanding consent depend on insufficient general health literacy remains to be examined. However, this may not be crucial to the existing practice viability in obtaining informed consent as a safeguard for respecting patients' autonomy in clinical trials. It is inevitable that patients recruited for clinical trials will have varying education and health literacy levels. There is no reason to assume that patients included in clinical trials have lower than average health literacy. Therefore, the outcomes suggest that, in the daily practice of clinical trials, patients with diverse education and health literacy levels agree to participate in medical testing based on defective, or at least incomplete, comprehension of the relevant information. This suggests that the present routines regarding patients' autonomy (and thus-dignity) in clinical trials are ethically questionable, if not explicitly flawed. 
None of the studies included in our review directly examined relations between patients' health literacy and their level of comprehension regarding the consent they granted. However, Chaisson et al. reported that patients' education was considered as a factor potentially influencing their understanding of the consent. They administered questionnaires in English or Setswana and concluded that participants who had a higher education level or chose to complete the questionnaires in English rather than Setswana demonstrated better overall comprehension. Similarly, Schumacher et al. and Krosin et al. found significant correlations between comprehension scores and formal education levels $[7,13]$. Ellis et al. distributed a survey to adult participants in the USA and Mali, plus the parents or guardians of a child in an additional group of Mali participants. Within Malian adults, only 9\% signed the IC, while the remaining $91 \%$ provided a fingerprint. In the Malian parents or guardians group, $84 \%$ provided a fingerprint rather than a signature. The literacy rate for parents or guardians varied between sites, ranging from 3 to $17 \%$. Of note, the questionnaire was initially intended to teach participants rather than to collect data [10]. The researchers found that patients' literacy was not a significant factor in their ability to understand the consent they were asked to grant.

The studies included in this review have some limitations that should be considered while interpreting the results. First, they demonstrated a high level of heterogeneity in sample size and type of underlying medical condition. We speculate that this may also have influenced patients' understanding of informed consent. For example, Pope et al. reported significant differences in the level of understanding within patients recruited to rheumatology, ophthalmology, and cardiology studies [15]. Similarly, Chu et al. reported that $61 \%$ of healthy controls were recruited from phase I trials, in which they were under the close monitoring and care of the researchers, while $80.8 \%$ of patients were recruited from phase III or IV trials that were conducted in outpatient clinics [8]. Second, especially in oncology, the presumed potential benefit of a novel treatment may exceed the presumed risk of the study, biasing patients towards consenting to participate in the trial despite a limited understanding of its experimental nature. Third, although education level was usually mentioned, health literacy was not examined in most studies. Additionally, other factors related to underlying disease may influence patients' comprehension, including fatigue, depression, cognitive status, and emotional factors associated with study inclusion and doctor's office visits. The resulting scope of interference with patients' ability to grasp the full meaning of the consent remains unexamined. However, such factors should be considered when assessing the current practice of obtaining informed consent. Finally, the scales used to examine patients' understanding differed across studies and ranged from multiple choice to Likertlike scale items, which limited our ability to adequately compare patients' understanding across studies.

In many cases, patients may be unaware that they lack understanding and therefore do not ask for clarification. In some cases, the information on expected therapeutic benefits may overshadow other aspects of the project, making patients less receptive to technical or more discouraging sides of the trial. Interestingly, our findings suggest that mothers asked to consent to including their children in a clinical trial were more determined to comprehend all relevant information than adult patients deciding on their own involvement in a trial. However, this is based on the results from a single study in this review [6].

The relatively consistent series of empirical findings opens further questions that have not been satisfactorily addressed in the literature to date. We hypothesise that patients seriously overrate their own level of comprehension. The extent to which they mistakenly feel that they have understood all relevant information while, in fact, they miss many important aspects of the consent present an interesting question that we are preparing to investigate. Similarly, physicians may seriously overrate their patients' level of comprehension based on (1) their own efforts to effectuate patients' understanding, (2) their confidence in patients' declaration of understanding and satisfaction, and (3) their own health literacy influencing their belief that the information offered to patients is easier to understand than is actually the case.

Thus, further research should target empirical testing of the hypothesised discrepancies between (1) the actual level of understanding by patients regarding what they consented to, (2) their subjective confidence that they understood what they consented to, and (3) physicians' confidence that their patients actually understood what they consented to.

Noting the scarcity of analogous research on the actual understanding of consent by patients in regular therapeutic practice, we recommend that future studies examine such comprehension in ordinary medical settings rather than only through the context of clinical trials. Since physicians typically take more care and effort to explain all relevant aspects of a clinical trial, we assume that, in a standard therapeutic setting, lack of comprehension regarding consent may be even larger. Therefore, a lack of relevant research in therapeutic clinical settings constitutes a remarkable gap in a crucial aspect of ethically viable medical practice.

\section{Conclusion}

We found that the level of comprehension regarding informed consent components, such as voluntary participation, blinding, and freedom to withdraw, was low, being understood by only half of the patients. This seriously undermines 
the ethical foundations of current practices for obtaining consent in clinical trials, potentially also challenging the standard approach to safeguarding patients' autonomy in ordinary medical settings.

\section{Abbreviations}

IC: Informed consent; IDU: Injection drug users; MEDLINE: Medical Literature analysis and retrieval system online (a database); MeSH: Medical subject headings; Embase: Excerpta Medica database

\section{Acknowledgements}

Not applicable.

\section{Authors' contributions}

T.S. conceived this study, decided on the framework for analysis, carried out the literature searching and analysis, and drafted the manuscript. K.S. decided on the framework for analysis, carried out the literature searching and analysis, and drafted the manuscript. All authors have read and approved the final version.

\section{Funding}

This research did not receive any specific grant from funding agencies in the public, commercial, or not-for-profit sectors.

\section{Availability of data and materials}

The datasets used and/or analysed during the current study are available from the corresponding author on reasonable request.

\section{Ethics approval and consent to participate}

Not applicable.

\section{Consent for publication}

Not applicable.

\section{Competing interests}

The authors declare that they have no competing interests.

\section{Author details}

${ }^{1}$ Research Centre for Public Policy and Regulatory Governance, Faculty of Law and Administration, University of Silesia in Katowice, Katowice, Poland. 2Department of Neurology; Centre of Expertise for Parkinson \& Movement Disorders, Radboud University Medical Center, Donders Institute for Brain, Cognition and Behaviour, PO Box 9101, 6500 HB Nijmegen, the Netherlands.

Received: 29 May 2020 Accepted: 11 December 2020

Published online: 14 January 2021

References

1. Agozzino E, Borrelli S, Cancellieri M, Carfora FM, Di Lorenzo T, Attena F. Does written informed consent adequately inform surgical patients? A cross sectional study. BMC Med Ethics. 2019;20(1):1.

2. Neff MJ. Informed consent: what is it? Who can give it? How do we improve it? Respir Care. 2008:53(10):1337-41.

3. Durand MA, Moulton B, Cockle E, Mann M, Elwyn G. Can shared decisionmaking reduce medical malpractice litigation? A systematic review. BMC Health Serv Res. 2015;15:167.

4. Manti S, Licari A. How to obtain informed consent for research. Breathe (Sheffield, England). 2018;14(2):145-52

5. Liberati A, Altman DG, Tetzlaff J, Mulrow C, Gotzsche PC, loannidis JP, et al. The PRISMA statement for reporting systematic reviews and meta-analyses of studies that evaluate healthcare interventions: explanation and elaboration. Bmj. 2009:339:b2700.

6. Minnies D, Hawkridge T, Hanekom W, Ehrlich R, London L, Hussey G. Evaluation of the quality of informed consent in a vaccine field trial in a developing country setting. BMC Med Ethics. 2008;9:15.

7. Schumacher A, Sikov WM, Quesenberry MI, Safran $H$, Khurshid $H$, Mitchell KM, et al. Informed consent in oncology clinical trials: a Brown University Oncology Research Group prospective cross-sectional pilot study. PLoS One. 2017;12(2):e0172957.
8. Chu SH, Jeong SH, Kim EJ, Park MS, Park K, Nam M, et al. The views of patients and healthy volunteers on participation in clinical trials: an exploratory survey study. Contemp Clin Trials. 2012;33(4):611-9.

9. Chaisson LH, Kass NE, Chengeta B, Mathebula U, Samandari T. Repeated assessments of informed consent comprehension among HIV-infected participants of a three-year clinical trial in Botswana. PLoS One. 2011; 6(10):e22696.

10. Ellis RD, Sagara I, Durbin A, Dicko A, Shaffer D, Miller L, et al. Comparing the understanding of subjects receiving a candidate malaria vaccine in the United States and Mali. Am J Trop Med Hyg. 2010;83(4):868-72.

11. Bergenmar M, Molin C, Wilking N, Brandberg Y. Knowledge and understanding among cancer patients consenting to participate in clinical trials. Eur J Cancer. 2008:44(17):2627-33.

12. Bertoli AM, Strusberg I, Fierro GA, Ramos M, Strusberg AM. Lack of correlation between satisfaction and knowledge in clinical trials participants: a pilot study. Contemp Clin Trials. 2007;28(6):730-6.

13. Krosin MT, Klitzman R, Levin B, Cheng J, Ranney ML. Problems in comprehension of informed consent in rural and peri-urban Mali, West Africa. Clin Trials. 2006;3(3):306-13.

14. Criscione LG, Sugarman J, Sanders L, Pisetsky DS, St Clair EW. Informed consent in a clinical trial of a novel treatment for rheumatoid arthritis. Arthritis Rheum. 2003:49(3):361-7.

15. Pope JE, Tingey DP, Arnold JM, Hong P, Ouimet JM, Krizova A. Are subjects satisfied with the informed consent process? A survey of research participants. J Rheumatol. 2003;30(4):815-24.

16. McNally T, Grigg J. Parents' understanding of a randomised double-blind controlled trial. Paediatr Nurs. 2001;13(4):11-4.

17. Itoh K, Sasaki Y, Fujii H, Ohtsu T, Wakita H, Igarashi T, et al. Patients in phase I trials of anti-cancer agents in Japan: motivation, comprehension and expectations. Br J Cancer. 1997;76(1):107-13.

18. Harrison K, Vlahov D, Jones K, Charron K, Clements ML. Medical eligibility, comprehension of the consent process, and retention of injection drug users recruited for an HIV vaccine trial. J Acquir Immune Defic Syndr Hum Retrovirol. 1995;10(3):386-90

19. Ponzio M, Uccelli MM, Lionetti S, Barattini DF, Brichetto G, Zaratin P, et al. User testing as a method for evaluating subjects' understanding of informed consent in clinical trials in multiple sclerosis. Mult Scler Relat Disord. 2018;25:108-11.

20. Page MJ, McKenzie JE, Higgins JPT. Tools for assessing risk of reporting biases in studies and syntheses of studies: a systematic review. BMJ Open. 2018:8(3):e019703.

21. Tam NT, Huy NT, Thoa le TB, Long NP, Trang NT, Hirayama K, et al. Participants' understanding of informed consent in clinical trials over three decades: systematic review and meta-analysis. Bull World Health Organ. 2015;93(3):186-98h.

22. Falagas ME, Korbila IP, Giannopoulou KP, Kondilis BK, Peppas G. Informed consent: how much and what do patients understand? Am J Surg. 2009; 198(3):420-35.

23. Idanpaan-Heikkila JE. WHO guidelines for good clinical practice (GCP) for trials on pharmaceutical products: responsibilities of the investigator. Ann Med. 1994:26(2):89-94.

\section{Publisher's Note}

Springer Nature remains neutral with regard to jurisdictional claims in published maps and institutional affiliations.

Ready to submit your research? Choose BMC and benefit from

- fast, convenient online submission

- thorough peer review by experienced researchers in your field

- rapid publication on acceptance

- support for research data, including large and complex data types

- gold Open Access which fosters wider collaboration and increased citations

- maximum visibility for your research: over $100 \mathrm{M}$ website views per year

At $\mathrm{BMC}$, research is always in progress.

Learn more biomedcentral.com/submissions 